\title{
Warm blood cardioplegia
}

Warm blood cardioplegia (WBC) was introduced for myocardial protection by the Toronto Group in the late 1980s. The term "normothermic blood cardioplegia" is more appropriate because the patient's temperature is maintained at $37^{\circ} \mathrm{C}$ throughout the operation by blood cardioplegia - anterograde, retrograde, or both.

Major advances in cardiac surgery and anaesthesia in the past three decades have combined to lower dramatically the risk to patients of all heart operations. The single most important development has been the introduction of cold crystalloid cardioplegia. Hypothermia was introduced into clinical practice by Bigelow and his colleagues in Toronto in 1950. Since then it has been the cornerstone of myocardial protection for most cardiac operations.

Despite its benefits, hypothermia has many drawbacks. At a biomolecular level, adenosine triphophatase (ATP) is thermolabile and its function is significantly impaired by hypothermia. This adversely affects all ATP-dependent reactions. These are many and include enzyme function, membrane stability, energy production, aerobic glucose utilisation, cyclic adenosine monophosphate production, and osmotic homoeostasis. Because hypothermia shifts the oxyhaemoglobin dissociation curve to the left, cold blood does not deliver oxygen to tissues in sufficient quantities, leading to hypoxia of already cold tissues. As a result, glucose is metabolised through the anaerobic Embden-Meyerhof pathway and lactate acidosis develops. This in turn leads to intracellular and, secondarily, extracellular falls in $\mathrm{pH}$, further impairing many enzymatic processes that are $\mathrm{pH}$ dependent. ${ }^{1}$ In addition, there is continuous myocardial deterioration during ischaemic time, albeit at a low rate, and reperfusion injury on release of the aortic cross clamp.

Electromechanical work is the major determinant of myocardial oxygen consumption. The ideal state of the heart during an operative procedure would be electromechanically arrested and perfused with blood-that is, in a state of aerobic arrest. This concept prompted the introduction of blood cardioplegia.

\section{Advantages}

The major advantages of blood as a cardioplegic perfusate are related to its ability to transfer oxygen to tissues, to buffer changes in $\mathrm{pH}$ appropriately, and to provide the appropriate osmotic environment for myocardial cells. All these major characteristics attributable to blood are optimal at normothermia and may be decreased, absent, or even deleterious as blood temperature is lowered: hence the advantage of normothermic blood cardioplegia over cold cardioplegia. Continuous normothermic blood cardioplegia (CNBC) not only prevents additional damage caused by ischaemia, hypothermia, and reperfusion but also, when the myocardium is at rest and provided with fundamental nutritional factors, it allows some degree of cellular repair during the operative period. ${ }^{2}$ Therefore aortic cross-clamping time is no longer equivalent to ischaemic time. This means that CNBC has a particular advantage in repair of complex cardiac anomalies, both congenital and acquired, which require hours of aortic cross clamping.

Many studies from different centres have confirmed the effectiveness of CNBC as a good method of myocardial protection with many advantages. All the studies so far have shown reduction of mortality in patients having CNBC, which was significant in those at high risk (NYHA class III and IV). The rates of intra-operative myocardial infarction are much lower. In most patients (up to $98 \%$ ) the heart starts beating spontaneously in sinus rhythm once the aortic cross clamp is removed. Most patients are weaned off cardiopulmonary bypass with little or no pharmacological or mechanical support. There is also immediate improvement in cardiac output in these patients, unlike hypothermic protection when cardiac output always falls in the immediate postoperative period. At the end of the operation all patients are warm and vasodilated when they arrive at the intensive therapy unit; this makes warming devices unnecessary and reduces the requirement for vasodilators. ${ }^{3-5}$ The patient is ready to be weaned off intermittent positive pressure ventilation much earlier. This may also be helped by the elimination of the risk of hypothermic injury of the phrenic nerve. In today's move towards "fast-tracking" postoperative care, this may prove to be a cost effective advantage over hypothermic techniques. During CNBC oxygen is delivered continuously and enzyme systems remain active. Therefore sufficient energy is produced to preserve the integrity of both the conduction systems and the myocardium. This is clearly demonstrated by low release of creatine kinase $\mathrm{MB}$ and a lower incidence of new and permanent conduction abnormalities which, when they occur, are usually transient. The incidence of postoperative supraventricular arrhythmias is not significantly reduced. ${ }^{6}$

There is less postoperative bleeding in patients who have CNBC, perhaps this is because vasodilated normothermic patients have a less acidic body environment when protamine is administered. Postoperatively the neurolopsychological function after normothermic cardiopulmonary bypass seems to be no worse than that after hypothermic procedures. ${ }^{7}$

\section{Problems}

Current clinical experience clearly shows that CNBC gives better myocardial protection and as a consequence improves the results of cardiac surgery. However, as with other methods of myocardial protection, there are problems. For the surgeon, the technique is cumbersome. There is poor visualisation of the surgical field in coronary artery bypass grafting, which means that 
cardioplegia may need to be interrupted while the distal anastomoses are inserted. Ischaemia at normal temperature increases the risk of myocardial stunning. We do not know for how long it is safe to interrupt cardioplegia. Nor do we know the optimal flow rates and pressures for the infusion of CNBC in patients with severe triple vessel coronary artery disease and a hypertrophied myocardium. The ideal composition of the cardioplegic solution is yet to be determined. It may need to be different from that of cold blood cardioplegia. Moreover, any accidental interruption of the cardiopulmonary bypass circuit makes a major central nervous system event more likely, because the patient's temperature is normal. $^{89}$ Clearly CNBC has more to offer cardiac surgeons, cardiologists, and their patients (particularly those in high risk groups with impaired left ventricular function and those requiring prolonged aortic crossclamp time). This promise can only be fulfilled if the problems associated with this exciting method of myocardial protection are solved. Then, and only then, will it find favour with more surgeons and be widely used.
Meanwhile further research and prospective randomised clinical studies are required to find solutions for these problems.

The Royal London Hospital,

A Y YOUHANA Whitechapel, London E1 1BB

1 Vaughan CC, Opie JC, Austin J. Warm blood cardioplegia. Ann Thorac Surg 1993;55:1227-32.

Lightenstein SV, Dalati HEL, Slutsky AS. Long cross-clamp time with warm heart surgery. Lancet 1989;i:1443.

3 Lichentenstein SV, Ashe KA, Slutsky AS. Warm heart surgery. 7 Thorac Cardiovasc Surg 1991;101:269-74.

4 Bottner RK, Wallace RB, Kent KM. Reduction of myocardial infarction after emergency coronary artery bypass grafting for failed coronary angioplasty with use of a normothermic reperfusion cardioplegia protocol. F Thorac Cardiovasc Surg 1991;101:1069-75.

5 The Warm Heart Investigators. Randomised trial of normothermic versus hypothermic coronary bypass surgery. Lancet 1994;343:559-63.

6 Flack JE III, Hafer J, Pekow P. Effect of normothermic blood cardioplegia on post-operative conduction abnormalities and supraventricular arrhythmias. Circulation 1992;86 (suppl II):385-92.

7 Wong BI, McLean RF, Fremes SE. Central nervous system dysfunction after warm or hypothermic cardiopulmonary bypass. Lancet 1992; 339:1383-4.

8 Matsurra H, Lazar HL, Shermin RJ. Warm versus cold blood cardioplegia-Is there a difference? $\mathcal{F}$ Thorac Cardiovasc Surg 1993;105:45-57.

9 Buckberg GD. Normothermic blood cardioplegia. Alternative or adjunct? $\mathcal{f}$ Thorac Cardiovasc Surg 1994;107:869-7. 